\title{
Kinematics modeling of a two DOFs continuum manipulator with uniform notches
}

\author{
Xiaolong Wang ${ }^{1}$, Haodong Wang ${ }^{1}$, Zhijiang $\mathrm{Du}^{1, *}$, and Wenlong Yang ${ }^{1}$ \\ ${ }^{1}$ State Key Laboratory of Robotics and System, Harbin Institute of Technology (HIT), Harbin, China
}

\begin{abstract}
Keywords: Kinematics, Mechanics, Continuum manipulator, Compliant joints, Single-port laparoscopy.

Abstract. Continuum manipulators have been widely adopted for single-port laparoscopy (SPL). A novel continuum manipulator with uniform notches which has two degrees of freedom (DOFs) is presented in this paper. The arrangement of flexible beams makes it own a higher load capacity. Its kinematic model is coupled with the mechanical model. The comprehensive elliptic integral solution (CEIS) is more practical in the actual deformation of the flexible beams. Based on that method, kinematics modeling is established from the driven space to the Cartesian space. The friction coefficient is an important factor which can affect the kinematic modeling. Therefore, an experimental platform is established to obtain the friction coefficient. The kinematic modeling is verified through the prototype. Experimental results show that the model has high precision.
\end{abstract}

\section{Introduction}

Robot design and control technology have made great progress recently. The traditional rigid manipulator can be used to complete the related tasks perfectly under the simple working environment of artificial construction. However, they cannot be used to complete relevant tasks well under the complex working environment, e.g. human body interior, complex pipelines, ruins, etc. The continuum manipulator is researched by more and more scholars to solve the problem of operations for complex space. The continuum manipulator is a kind of bionic mechanism, which is inspired by the biological structures, e.g. elephant trunk, snake and tentacles. The continuum manipulator, which has more freedom and better bending characteristics can be used to complete complex operations in a narrow space. It can be classified into discrete and continuum configurations. The discrete configuration is similar to the traditional rigid manipulator, which is formed by a series connection of multiple modular joints and supported by the elastic body. The continuum configuration can be regarded as being formed by the series connection of the flexible mechanism. The complete and uninterrupted elastic material can be taken as the skeleton of the continuum configuration directly. It not only owns no gap, high precision characteristics [1], but also has

\footnotetext{
*Corresponding author: duzj01@hit.edu.cn
} 
better flexibility and biocompatibility, which can help it complete more flexible movement inside the human body, and do less damage to the internal tissues of the human body.

As a typical representative of minimally invasive surgery (MIS), laparoscopic surgery generally requires opening 2-4 minimally invasive holes on the surface of the human body. SPL appeared in order to minimize the risk of such trauma and infection. The flexibility and small size of the continuum manipulator make it possible to be applied in SPL. Roh et al. have developed an SPS robot system that transmits motion through a saddle-shaped surface with end loads up to $1 \mathrm{kgf}$ [2]. Choset et al. designed a Cardio ARM robot system which is made up of rigid cylindrical links in series [3]. Li et al. developed a CTSM robot that transmits motion through a spherical surface, and it's tested currently [4]. The above examples are the discrete configuration. In contrast to it, there are some examples of continuous configuration. Ding et al. developed the IREP robot system based on a flexible continuum manipulator which is made of nickel-titanium memory alloy [5]. Kutzer et al. developed a CDM flexible arm based on a notched continuum [6]. Xu et al. developed an SJTU unfoldable robotic system that can pass the $\Phi 12 \mathrm{~mm}$ hole [7]. Du et al. have developed two single-hole surgical robots based on different forms of notches [8,9].

The above examples demonstrate the feasibility and effectiveness of the continuum manipulator applied in single-hole surgery. It is necessary to choose a suitable modeling method to improve the control accuracy of the manipulator. The deformation of the continuum configuration manipulator is related to the end load. Yang established a mechanical model through a cantilever beam theory which is suitable for small linear elastic deformation [8]. Yang established a mechanical model through a Timoshenko beam theory which is suitable for small linear elastic deformation [9]. The shear and rotation effects are considered in the Timoshenko beam theory. Gao et al. proposed a modeling method based on Cosserat rod theory which is suitable for elastic rods with super large deformation [10]. The shear deformation, centerline expansion and deformation, and bulk force effects are considered in Cosserat rod theory.

The main problem of continuum configuration is lower load capacity. In order to improve its practicability in SPL, more scholars have invested in research to improve its load capacity. In this paper, a uniform notch continuum with a strong load capacity is proposed. The design and characters of the structure are introduced in Section 2. The kinematic modeling method based on CEIS is presented in Section 3. The accuracy of the kinematic model can be proved by experiments in section 4 .

\section{Structural design}

In this paper, a continuum manipulator with uniformed notches is obtained by the superelasticity of Nitinol. Nitinol tube's length is $142 \mathrm{~mm}$, outer diameter is $10 \mathrm{~mm}$, and inner diameter is $8.8 \mathrm{~mm}$. The notches are obtained by wire-cut electrical discharge machining (WEDM). The continuum manipulator proposed in this paper adopts an elliptical straight beam notch scheme, as shown in Fig. 1(a).

$A$ and $B$ are two fixed points on the continuum manipulator. When they are located, the ellipse can be independently adjusted for beam length $l$ and thickness $h$. It is beneficial for further optimization of the structure. Besides, the ellipse can be used to reduce stress concentration.

The bending planes of adjacent flexible beams are perpendicular to each other, as shown in Fig. 1(b), (c). Another arrangement is to place flexible beams with the same bending direction together, as shown in Fig. 1(d). In Fig. 1(c), (d), flexible beam m represents the beam which is closest to the base among the beams whose bending plane is plane x-z. When the end of the continuum manipulator is subjected to a load $F$, the bending angle of beam $\mathrm{m}$ 
in Fig. 1(c) is larger than that in Fig. 1(d). This can increase the range of motion of the continuum manipulator in the plane x-z. Therefore, the arrangement of Fig. 1(c) is adopted in this paper.
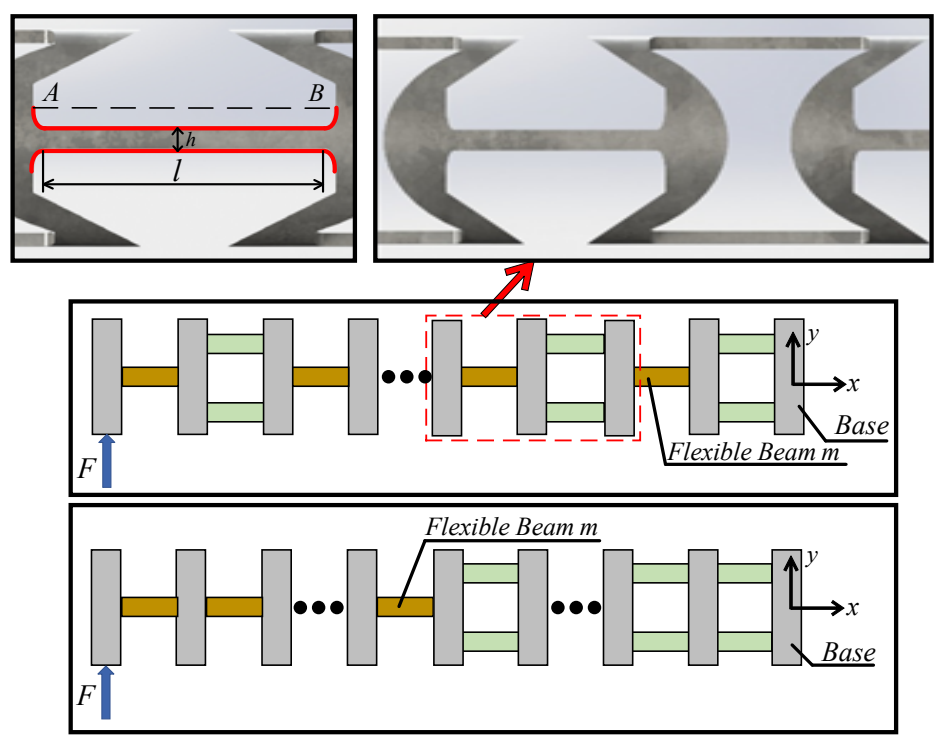

Fig. 1. (a) Notch shape. (b) Arrangement of flexible beams. (c) The bending planes of adjacent flexible beams are perpendicular. (d) The bending planes of adjacent flexible beams are the same.

The cross-section of the flexible beam $i$ is shown in Fig. 2. The moment of inertia of the section to the neutral axis can be obtained as follows:

$$
I_{z i}=\int y_{\varphi}^{2} d A=\frac{r_{2}^{4}}{2} \arcsin \frac{h_{i}}{2 r_{2}}-\frac{r_{2}^{3} h_{i}}{4}\left(1-\frac{h_{i}^{2}}{2 r_{2}^{2}}\right) \sqrt{1-\frac{h_{i}^{2}}{4 r_{2}^{2}}}-\frac{r_{1}^{4}}{2} \arcsin \frac{h_{i}}{2 r_{1}}+\frac{r_{1}^{3} h_{i}}{4}\left(1-\frac{h_{i}^{2}}{2 r_{1}^{2}}\right) \sqrt{1-\frac{h_{i}^{2}}{4 r_{1}^{2}}}
$$

where $I z i$ is the moment of inertia of the section of flexible beam $i$ transversely facing the neutral axis $z_{i}, r_{1}$ is the inner diameter of the nitinol tube, $r_{2}$ is the outer diameter of the nitinol tube, and $h_{i}$ is the thickness of flexible beam $i$.

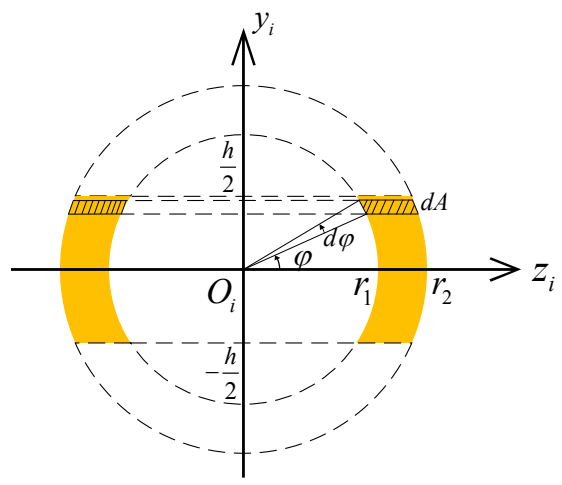

Fig. 2. Cross-section of the flexible beam $i$. 


\section{Kinematics model}

The deflection of some flexible beams exceeds $10 \%$ of the beam length, which is a large deflection deformation. In order to describe this deformation more accurately, the relationship between the load and deformation is described through CEIS in this paper [11]. The mechanics model is briefly introduced in Subsection 3.1. Since the kinematics modeling is inseparable from the mechanical model, the process of solving the end load of each flexible beam is mainly introduced in Subsection 3.2. Through combining the work of the first two sections, the position and pose of the continuum end are solved in Subsection 3.3.

\subsection{Mechanics model}

Each flexible beam can be regarded as a cantilever beam model. Load parameters, structure parameters, and deformation parameters are shown in Fig. 3. The coordinate system $\left\{i_{-} 1\right\}$ is attached to the forced end of the flexible beam. The coordinate system $\left\{i_{-} 2\right\}$ is attached to the fixed end of the flexible beam. In the figure, $a_{i}$ and $b_{i}$ represent the change in position of the end, and $\theta_{o i}$ represents the change in pose of the end.

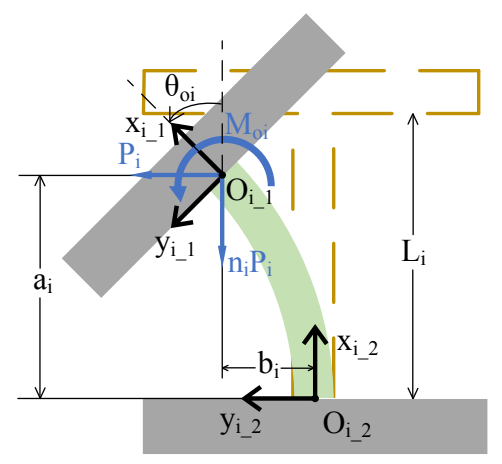

Fig. 3. Flexible beam deformation diagram.

The deformation parameters of the flexible beam can be obtained by iterating the following two sets of expressions:

$$
\left.\begin{array}{c}
\alpha=\frac{S_{M} \sqrt{2}}{\sqrt{\lambda+\eta}} f \\
\frac{a_{i}}{L_{i}}=\frac{S_{M} \sqrt{2}}{\eta^{2} \alpha \sqrt{\lambda+\eta}}\left[-n_{i} \lambda f+n_{i}(\lambda+\eta) e+\sqrt{\lambda+\eta} c\right] \\
\frac{b_{i}}{L_{i}}=\frac{S_{M} \sqrt{2}}{\eta^{2} \alpha \sqrt{\lambda+\eta}}\left[\lambda f-(\lambda+\eta) e+n_{i} \sqrt{\lambda+\eta} c\right]
\end{array}\right\}
$$


The algorithm flow chart is shown in Fig. 4. An inflection point is a point where the curvature is zero. In the figure, $m$ indicates the number of inflection points. When load parameters and structure parameters are known, deformation parameters can be obtained through the algorithm.

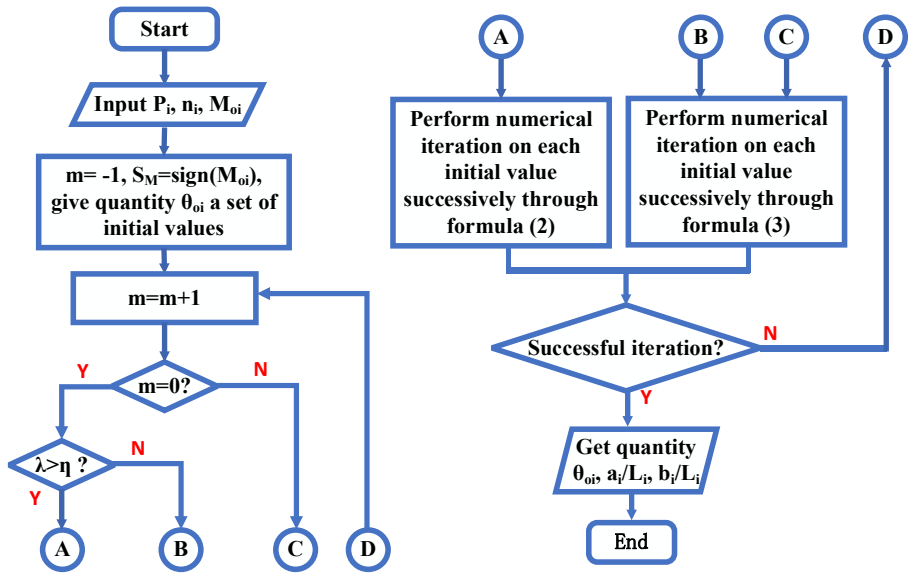

Fig. 4. Algorithm flow for solving deformation parameters of the flexible beam.

\subsection{Solution for the deformation parameters of each flexible beam}

The flexible beam $i$ is placed between the rigid body $i$ and the rigid body $i+1$, as shown in Fig. 5 . The rules of establishing the coordinate system are the same as those of the mechanical model [see Fig. 3]. The coordinate system $\left\{i_{-} 1\right\}$ is attached to the contact surface between the flexible beam $i$ and rigid body $i$. The coordinate system $\left\{i_{-} 2\right\}$ is attached to the contact surface between the flexible beam $i$ and rigid body $i+1$. Similarly, the coordinate system $\left\{i-1 \_1\right\}$ is attached to the contact surface between the flexible beam $i-1$ and rigid body $i-1$. The coordinate system $\left\{i-1 \_2\right\}$ is attached to the contact surface between the flexible beam $i-1$ and rigid body $i$. Therefore, two coordinate systems $\left\{i-1 \_2\right\}$ and $\left\{i \_1\right\}$ are both fixed in the rigid body $i$. Each element of the homogeneous transformation matrix $\underset{i-1-1-2}{i-1} T$ is constant.

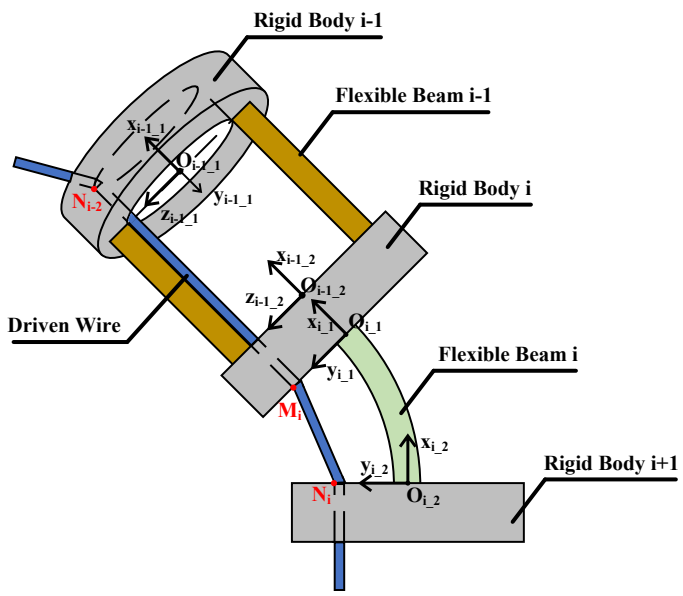

Fig. 5. The schematic diagram for the deformation of the flexible beam $i$. 
According to the parity of $i$, the homogeneous transformation matrix ${ }_{i-1}^{i-}{ }_{-}{ }_{2}^{1} T$ can be obtained as follows:

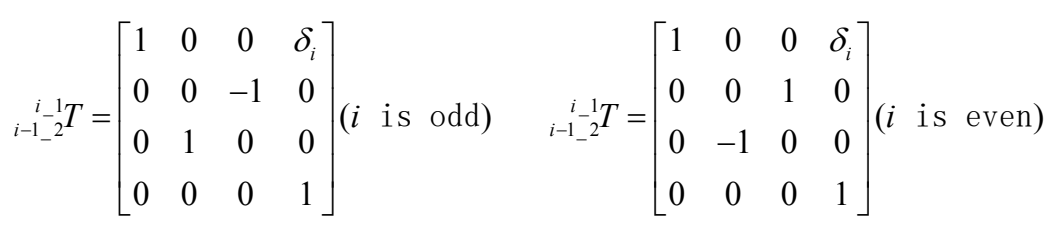

where $\delta_{i}$ is the $x_{i_{-} l}$ component of the vector $\boldsymbol{O}_{i_{-} 1} \boldsymbol{O}_{\boldsymbol{i - 1}{ }_{-} 2}$, which is 0 in this paper.

The initial values $a_{i}, b_{i}$ and $\theta_{o i}$ are $L_{i}, 0$ and 0 , respectively. From Fig. 3, the homogeneous transformation matrix of $\left\{i_{-} 1\right\}$ with respect to $\left\{i_{-} 2\right\}$ can be observed as

$$
{ }_{i_{-}-1}^{i_{-}} T=\left[\begin{array}{cccc}
\cos \theta_{o i} & -\sin \theta_{o i} & 0 & a_{i} \\
\sin \theta_{o i} & \cos \theta_{o i} & 0 & b_{i} \\
0 & 0 & 1 & 0 \\
0 & 0 & 0 & 1
\end{array}\right] \text {. }
$$

A driven wire can be divided into many sections small driven wires. When the forces provided by driven wires outside the plane $y_{i_{-}} z_{i_{-} l}$ are known, the end-load of the flexible beam $i$ can be solved. Only the forces provided by driven wire $N_{i-2} M_{i}$ are unknown before this iteration. The equivalent force vectors of other known forces are stored in the matrix $F_{V D}$. The matrix $F_{V D}$ is a matrix of $3 \times(2 \mathrm{i}-4)$, and each column vector corresponds to an external force vector in the coordinate system $\left\{i-1 \_2\right\}$. The equivalent origins of these known forces are stored in the matrix $F_{p o}$. The matrix $F_{p o}$ is a matrix of $4 \times(2 \mathrm{i}-4)$, and each column vector corresponds to a homogeneous coordinate of an origin in the coordinate system $\left\{i-1 \_2\right\}$. The expressions of $F_{V D}$ and $F_{p o}$ in the coordinate system $\left\{i_{-} 2\right\}$ are shown as

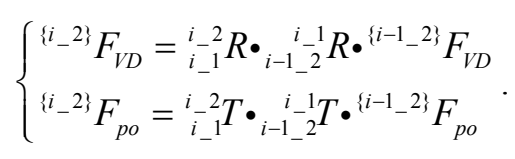

Before this iteration, ${ }^{\{i-1}{ }_{-}^{2\}} N_{i-2}$ is a known quantity, and its expression in the coordinate system $\left\{i_{-} 2\right\}$ is shown as

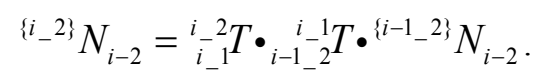

As $i>2$, the friction forces between the driven wires and continuum manipulator are considered in this paper. There are three assumptions as follows:

1) The Coulomb friction model is applied, ie $\left|F_{f_{-} i n}\right|=\mu\left|F_{N i_{-} i n}\right|$, where $F_{f_{-} \text {in }}$ and $F_{N_{-} i n}$ are the friction force and the normal force provided by the flexible beam $i-1$, respectively;

2) The driven wires pass through $N_{i-2}$ and $M_{i} . N_{i-2}$ is a fixed point on the rigid body $i-1$, and $M_{i}$ is a fixed point on the rigid body $i$;

3) The driven wires are only subjected to the force at $N_{i-2}$ and $M_{i}$. The direction of normal force lies in the bisector of two driven wires. The direction of the friction force is perpendicular to that of the normal force.

In order to obtain the forces provided by the driven wire $N_{i-2} M_{i}$ on the flexible beam $i-1$, the force analysis is carried out. Taking the driven wire $N_{i-2} M_{i}$ as the research object, the 
force analysis diagram is shown in Fig. 6. There are three parts in external forces applied on the driven wire $N_{i-2} M_{i}$ :

1) $F_{T_{-}} N_{i-2} M_{i-2}$ is the tensile force provided by the driven wire $M_{i-2} N_{i-2}$, the direction is from $N_{i-2}$ to $M_{i-2}$, and the magnitude is known before this iteration;

2) $F_{T_{-}} M_{i} N_{i}$ is the tensile force provided by the driven wire $M_{i} N_{i}$, the direction is from $M_{i}$ to $N_{i}$, and the magnitude is unknown before this iteration;

3 ) The friction forces and normal forces provided by the flexible beam $i-1$. The specific directions of friction forces are related to the deformation trend of the flexible beam $i-1$ [see Fig. 6(a), (b)]. Therefore, the specific directions of friction forces need to be discussed. The magnitudes of the normal forces and the friction forces are unknown.

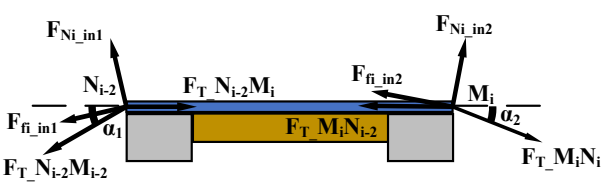

(a)

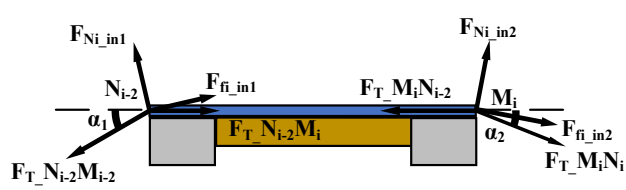

(b)

Fig. 6. The external forces applied on the wire rope. (a) The flexible beam is deforming. (b) The flexible beam is returning to its original state.

Through the force balance equation, the magnitude of tension $F_{T_{-}} M_{i} N_{i}$ can be obtained as follows:

$$
\left\{\begin{array}{l}
\left|F_{T_{M_{i} N_{i}}}\right|=\left|F_{T_{N_{i-2} M_{i-2}}}\right| \cdot \frac{\left(\cos \frac{\alpha_{1}}{2}+\mu \sin \frac{\alpha_{1}}{2}\right)\left(\cos \frac{\alpha_{2}}{2}+\mu \sin \frac{\alpha_{2}}{2}\right)}{\left(\cos \frac{\alpha_{1}}{2}-\mu \sin \frac{\alpha_{1}}{2}\right)\left(\cos \frac{\alpha_{2}}{2}-\mu \sin \frac{\alpha_{2}}{2}\right)} \text { Beam is deforming } \\
\left|F_{T_{M_{i} N_{i}}}\right|=\left|F_{T_{N_{i-2} M_{i-2}}}\right| \cdot \frac{\left(\cos \frac{\alpha_{1}}{2}-\mu \sin \frac{\alpha_{1}}{2}\right)\left(\cos \frac{\alpha_{2}}{2}-\mu \sin \frac{\alpha_{2}}{2}\right)}{\left(\cos \frac{\alpha_{1}}{2}+\mu \sin \frac{\alpha_{1}}{2}\right)\left(\cos \frac{\alpha_{2}}{2}+\mu \sin \frac{\alpha_{2}}{2}\right)} \text { Beam is returning }
\end{array}\right.
$$

where $\alpha_{1}=\arccos \left(\frac{N_{i-2} M_{i-2} \cdot M_{i} N_{i-2}}{\left|N_{i-2} M_{i-2}\right| \cdot\left|M_{i} N_{i-2}\right|}\right), \alpha_{2}=\arccos \left(\frac{N_{i-2} M_{i} \bullet M_{i} N_{i}}{\left|N_{i-2} M_{i}\right| \cdot\left|M_{i} N_{i}\right|}\right)$.

The friction forces and normal forces provided by the driven wire $N_{i-2} M_{i}$ can be replaced by $F_{T_{-}} N_{i-2} M_{i-2}$ and $F_{T_{-}} M_{i} N_{i}$ equivalently. Therefore, what are actually stored in the $F_{V D}$ are the tensile forces provided by different driven wires. Considering that the effect of interaction forces between adjacent driven wires can be offset, the end-load of the flexible beam $i(i>2)$ can be simplified as follows:

$$
\left\{\begin{array}{l}
{ }^{\left\{i_{-} 2\right\}} F_{i}={ }^{\left\{i_{-} 2\right\}} F_{T_{M_{i-1} N_{i-1}}}+{ }^{\left\{i_{-}-2\right\}} F_{T_{M_{i} N_{i}}} \\
{ }^{\left\{i_{-} 2\right\}} M_{O_{i_{-}}}\left(F_{i}\right)={ }^{\left\{i_{-} 2\right\}} O_{i_{-} 1} M_{i-1} \times{ }^{\left\{i_{-} 2\right\}} F_{T_{M_{i-1} N_{i-1}}}+{ }^{\left\{i_{-} 2\right\}} O_{i_{-} 1} M_{i} \times{ }^{\left\{i_{-} 2\right\}} F_{T_{M_{i} N_{i}}}
\end{array}\right.
$$

where $F_{T_{-}} M_{i-1} N_{i-1}$ is the tensile force provided by the driven wire $M_{i-1} N_{i-1}$, and it's known before this iteration.

The algorithm flow for solving the deformation parameters of the flexible beam $i(i>2)$ is shown in Fig. 7. 

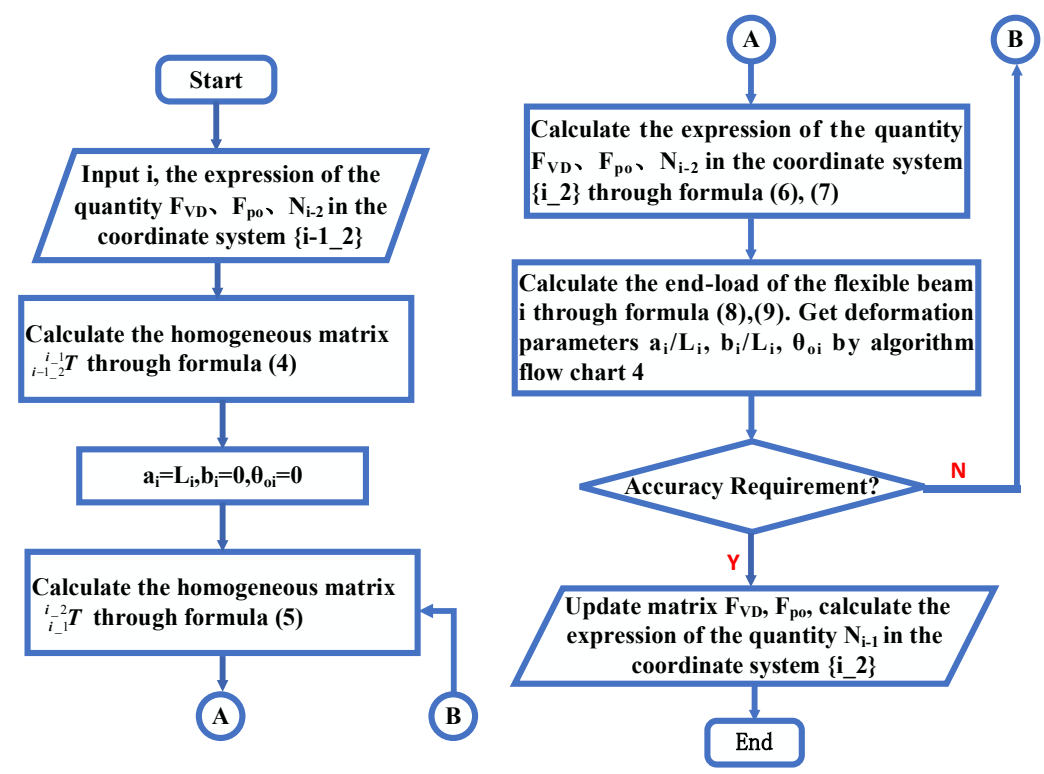

Fig. 7. Algorithm flow for solving deformation parameters of the flexible beam $i$.

The solution process of the first two flexible beams is similar to the above process. Since one end of the first driven wire is fixed on the rigid body 1 , there is no friction force between the first driven wire and the rigid body 1 . Similarly, there is no friction force between the second driven wire and the rigid body 2 . Therefore, friction forces don't exist during the solution process of the first two flexible beams. The end load of the flexible beam $i$ can be simplified as follows:

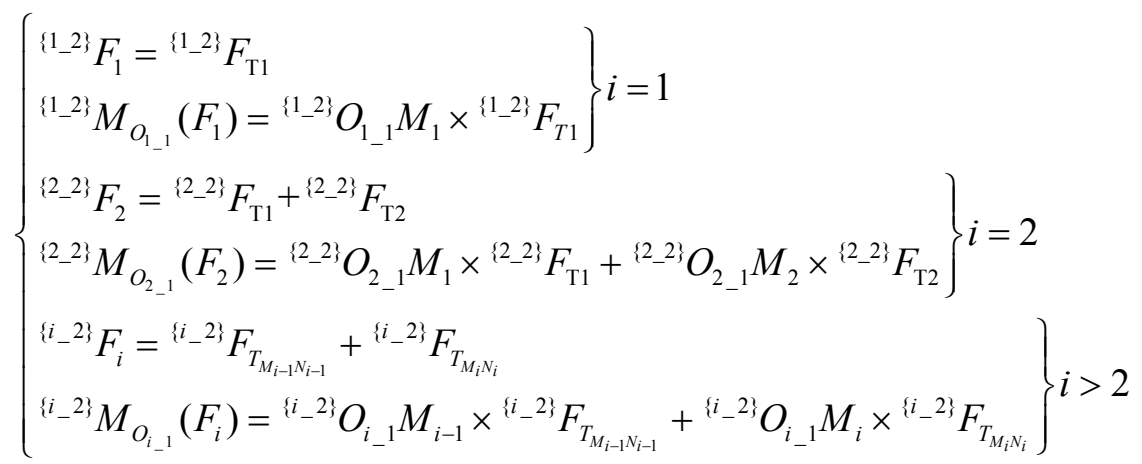

where $F_{T 1}$ is the tensile force provided by the first driven wire on the rigid body 1 , and $F_{T 2}$ is the tensile force provided by the second driven wire on the rigid body 2 .

\subsection{Solving for position and pose of continuum end}

After solving the deformation parameters of each flexible beam, the homogenous matrix from the tip to the base is shown as

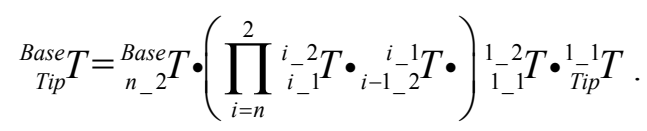


The operating space is shown in Fig. 8. As can be seen from the figure, the operating space can meet general SPL needs.

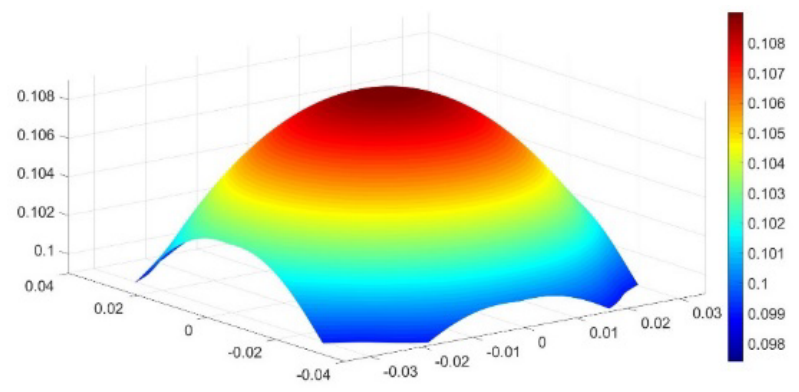

Fig. 8. The operating space.

\section{Experiments and results}

The kinematics model is verified through experiments in this section. An experimental platform is established to obtain the friction coefficient in Subsection 4.1. The verification experiment of the kinematics model is introduced in Subsection 4.2.

\subsection{Measurement experiment for friction coefficient}

The schematic diagram of the experimental model is shown in Fig. 9(a). Before the start of each set of experiments, the angle $\alpha_{1}$ is known. Then the angle $\alpha_{2}$ is adjusted by the ball screw until the system reaches the critical friction state. The friction coefficient can be obtained as

$$
\mu=\frac{-\left(m_{1}+m_{2}\right) \sin \frac{\alpha_{1}+\alpha_{2}}{2}+\sqrt{\left(m_{1}+m_{2}\right)^{2} \sin ^{2} \frac{\alpha_{1}+\alpha_{2}}{2}-\left(m_{1}-m_{2}\right)^{2} \sin \alpha_{1} \sin \alpha_{2}}}{2\left(m_{1}-m_{2}\right) \sin \frac{\alpha_{1}}{2} \sin \frac{\alpha_{2}}{2}} .
$$

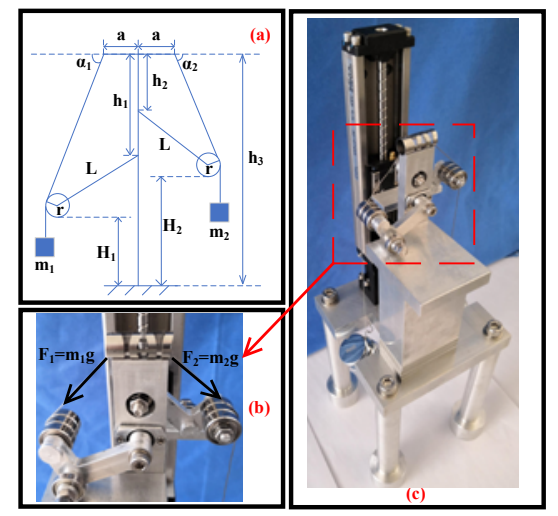

Fig. 9. Experiment for Friction Coefficient. (a) Experimental principle diagram. (b) Partially enlarged view. (c) The overall view of the experimental bench.

The physical quantities of the formula are shown in the schematic diagram. The partially enlarged view and the overall view of the experimental bench are shown in Fig. 9(b) and (c), 
respectively. 18 sets of measurement experiments are carried out. The mean value is taken as the friction coefficient, which is 0.3117 [see Fig. 10].

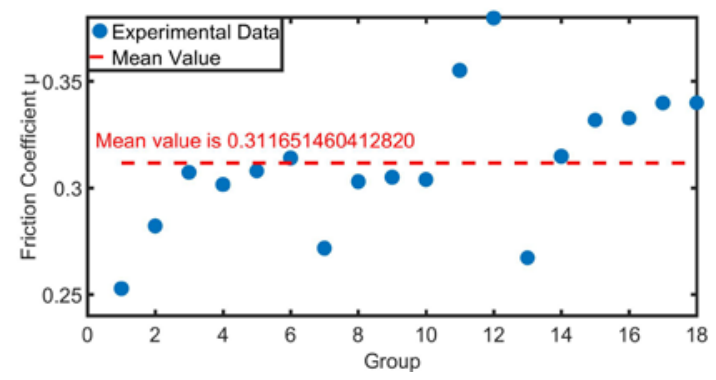

Fig. 10. Experimental data and result.

\subsection{Validation of kinematics model}

The experiment platform for validation of the kinematics model is shown in Fig. 11(a). The continuum manipulator is located in the fixed table. The end of the manipulator is pulled by the weight through the driven wire. The manipulator begins to deform under the tensile force of the weight. The photos of deformation results could be taken by the CCD camera, as shown in Fig. 11(b).

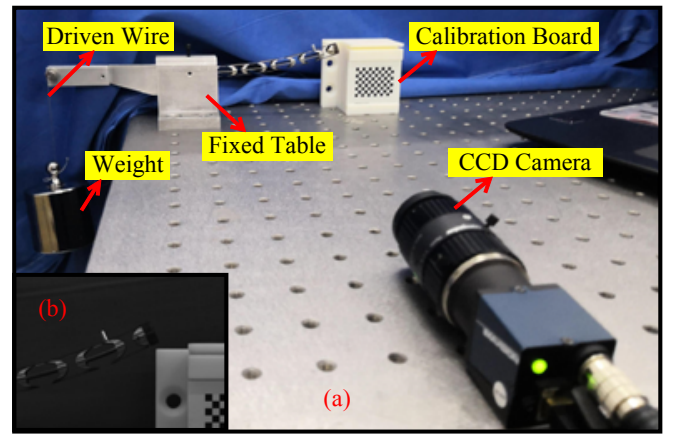

Fig. 11. Experiment for validation of the kinematics model. (a) Experiment platform. (b) The photo got by the CCD camera.

During the experiment, the load is increased from 0 to $1.5 \mathrm{~kg}$ in increments of $0.1 \mathrm{~kg}$. Through the calibration board, the position of the tip end of the manipulator could be calculated by Matlab. The results of the experimental and theoretical values are shown in Fig. 12(a). The error of each experimental result is shown in Fig. 12(b). The results indicate that the mean tip error of all the verification experiments is $0.597 \pm 0.556 \mathrm{~mm}$. The max tip error is $1.153 \mathrm{~mm}$, which is less than $5 \%$ of the total manipulator length. The possible sources are the gravity of the manipulator, gravity acceleration value, and image acquisition. 

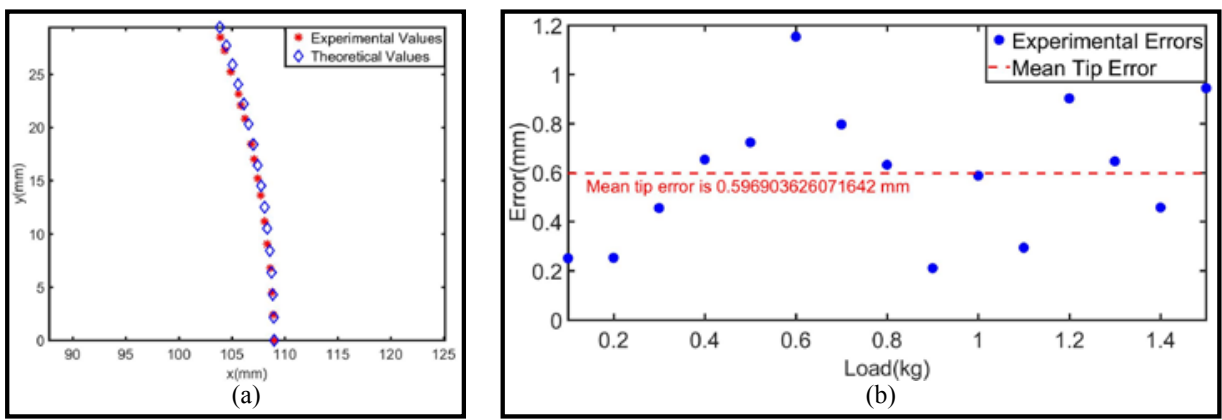

Fig. 12. (a) The results of the experimental and theoretical values. (b) The errors of all the experimental results.

\section{Summary}

A two DOFs continuum manipulator with uniform notches is proposed in this paper. The kinematics model and the mechanical model are coupled. The mechanical model is established through CEIS. The tensile forces, normal forces and friction forces provided by driven wires are considered to establish the kinematics model. The friction coefficient is measured through a specific experimental platform. The results indicate that the mean tip error of all the verification experiments is $0.597 \pm 0.556 \mathrm{~mm}$. All the errors are less than $5 \%$ of the total manipulator length. A future study could focus on the solution of inverse kinematics, force feedback and shape perception.

This research was supported in part by the National Natural Science Foundation of China under Grant 61673139, Grant U1813213 and Heilongjiang Science Foundation under Grant JC2016014.

\section{References}

[1] Larry L. Howell, Spencer P. Magleby, Brian M. Olsen, et al., "Handbook of Compliant Mechanisms," John Wiley \& Sons Ltd, Hoboken, 2013.

[2] Roh K S, Yoon S, Kwon Y D, et al. "Single-Port Surgical Robot System with Flexible Surgical Instruments," International Conference on Intelligent Robotics and Applications. Springer, Cham, 2015:447-459.

[3] Rivera-Serrano C M, Johnson P, Zubiate B, et al., "A transoral highly flexible robot," Laryngoscope, 2012, 122(5):1067-1071.

[4] Zheng Li, Haoyong $\mathrm{Yu}$, et al. "A Novel Constrained Tendon-driven Serpentine Manipulator," IEEE Robotics and Automation Letters, 2015.

[5] J. Ding, R. E. Goldman, K. Xu, P. K. Allen, D. L. Fowler, and N. Simaan, "Design and coordination kinematics of an insertable robotic effectors platform for single-port access surgery," IEEE/ASME Trans. Mechatronics, vol. 18, no. 5, pp. 1612-1624, Oct. 2013.

[6] M. D. Kutzer et al., "Design of a new cable-driven manipulator with a large open lumen: Preliminary applications in the minimally-invasive removal of osteolysis," in Proc.

IEEE Int. Conf. Robot. Autom., Shanghai, China, 2011, pp. 2913-2920

[7] Kai Xu, et al., " Development of the SJTU Unfoldable Robotic System (SURS) for Single-Port Laparoscopy," IEEE/ASME Transactions on Mechatronics, vol. 20, no. 5, Oct. 2015. 
[8] Z X Yang, et al., "Kinematics Modelling of Tendon-Driven Continuum Manipulator with Crossed Notches," International Conference on Robotics and Mechatronics, 2017.

[9] Wenlong Yang, et al., "Kinematics Modeling for a Kinematic-Mechanics Coupling Continuum Manipulator," International Conference on Manipulation, Oct 2014.

[10] Anzhu Gao, et al., "Mechanical Model of Dexterous Continuum Manipulators With Compliant Joints and Tendon/External Force Interactions," IEEE/ASME Transactions on Mechatronics, vol. 22, no. 1, Feb. 2017.

[11] Aimei Zhang, Guimin Chen. "A Comprehensive Elliptic Integral Solution to the Large Deflection Problems of Thin Beams in Compliant Mechanisms," Transactions of the ASME, Journal of Mechanisms and Robotics,2013,5(2):021006. 